\title{
The Effects of Ramelteon on Glucose Metabolism and Sleep Quality in Type 2 Diabetic Patients With Insomnia: A Pilot Prospective Randomized Controlled Trial
}

\author{
Tetsuji Tsunoda a, b, d , Masayo Yamada ${ }^{a}$, Tomoaki Akiyama ${ }^{a}$, Taichi Minami ${ }^{a}$, Taishi Yoshii ${ }^{b}$, \\ Yoshinobu Kondo ${ }^{\text {b, c }}$, Shinobu Satoh ${ }^{c}$, Yasuo Terauchi ${ }^{\mathrm{b}}$
}

\begin{abstract}
Background: Insomnia is associated with the onset and development of diabetes. Melatonin affects sleep quality and glucose metabolism in diabetic patients with insomnia. We administered ramelteon, an agonist of melatonin, to type 2 diabetic patients and investigated its effects on glucose metabolism and insomnia.

Methods: This multicenter, prospective, randomized, and observational pilot study was performed between April 2014 and April 2015 at three institutes in Japan. Patients were prescribed ramelteon $8 \mathrm{mg} /$ day for 3 months (first period). And patients were divided at random into the continuation group that continued taking ramelteon and the discontinuation group that discontinued taking ramelteon for 3 additional months (second period). The primary endpoint was change in glycated hemoglobin (HbA1c) level. Secondary endpoints were changes in global Pittsburgh sleep questionnaire index (PSQI) score and other glucose metabolism makers.
\end{abstract}

Results: We enrolled 42 patients, and 32 patients completed the first period. Their mean HbAlc was $6.7 \%$, and global PSQI score was 8.1 on average. HbA1c level did not change but global PSQI score improved from 8.1 to 7.2 by ramelteon $(\mathrm{P}=0.030)$. Thirty-one patients completed the second period. HbAlc level did not change in the continuation group, but it increased from $6.7 \%$ to $6.9 \%(\mathrm{P}=0.003)$ in the discontinuation group. Global PSQI score did not change in each group. There was no rebound insomnia.

Conclusion: Treatment with ramelteon did not change the HbAlc

Manuscript accepted for publication September 28, 2016

aDivision of Metabolism and Endocrinology, Department of Internal Medicine, Yokohama Sakae Kyosai Hospital, Federation of National Public Service Personnel Mutual Associations, Yokohama, Japan

bDepartment of Endocrinology and Metabolism, Graduate School of Medicine, Yokohama City University, Yokohama, Japan

'Department of Endocrinology and Metabolism, Chigasaki Municipal Hospital, Chigasaki, Japan

${ }^{\mathrm{d}}$ Corresponding Author: Tetsuji Tsunoda, Division of Metabolism and Endocrinology, Department of Internal Medicine, Yokohama Sakae Kyosai Hospital, Federation of National Public Service Personnel Mutual Associations, 132 Katsura-cho, Sakae-ku, Yokohama 247-8581, Japan.

Email: tsunoda9939@gmail.com

doi: http://dx.doi.org/10.14740/jocmr2754w level but improved sleep quality in type 2 diabetic patients with insomnia. Discontinuation of ramelteon slightly increased the HbAlc level and did not worsen sleep quality.

Keywords: Type 2 diabetes mellitus; Insomnia ramelteon; Pittsburgh sleep questionnaire index; Short form 36

\section{Introduction}

Insomnia is frequently observed in patients with diabetes [1]. Insomnia leads to insulin resistance and promotes obesity because insomnia impairs daytime activities of daily living (ADL), and decreases leptin secretion while increasing ghrelin secretion $[2,3]$. Insomnia is also a cause of oxidative stress [4]. Insomnia with short sleep duration is reportedly associated with increased odds of diabetes [5]. Therefore, insomnia is associated with diabetes. Besides, the long-term persistence of changes in the living environment and aging lead to decreased melatonin secretion [6-8].

Although a main role of the neurohormone melatonin is its participation in the adjustment of sleep, a decrease in melatonin is a risk factor for the onset of diabetes [9]. Recently, various research findings regarding the effects of melatonin on glucose metabolism have been reported. Namely, insulin resistance has been observed in pinealectomized rats [10]. Blood melatonin level was found to be lower in patients with diabetes [11]. Furthermore, melatonin improved insulin resistance in type 2 diabetic rats and glycated hemoglobin $(\mathrm{HbA} 1 \mathrm{c})$ levels in type 2 diabetic patients [12-14].

In Japan, the Pharmaceutical Affairs Law has banned the sale of melatonin itself, including supplement forms. However, ramelteon, a melatonin agonist, was approved for patients with insomnia in 2010 in Japan [15]. Ramelteon is currently under investigation as an alternative to conventional gammaaminobutyric acid receptor A (GABAA) activators. Ramelteon has a 5.8-fold higher affinity for melatonin MT1 receptor and a 3.4-fold higher affinity for melatonin MT2 receptor compared with melatonin [16]. Although there have been reports on the effect of melatonin on glucose metabolism [12, 17, 18], that of ramelteon has not been discussed. In addition, the efficacy and safety of ramelteon in patients with type 2 diabetes are yet to be researched. 
These conditions inspired us to investigate the effects of ramelteon in type 2 diabetic patients with insomnia.

\section{Materials and Methods}

\section{Study design}

This pilot study was performed as a multicenter, prospective, randomized, and observational study between April 2014 and April 2015 at three institutes in Japan: Yokohama Sakae Kyosai Hospital, Chigasaki Municipal Hospital, and Yokohama City University Hospital. The study was approved by the ethics committee of each institute. This study was registered with the UMIN Clinical Trial Registry (UMIN 000010074).

\section{Subjects}

Type 2 diabetic patients with untreated chronic insomnia who regularly visited the hospital were assessed for eligibility for this study. Patients were included if their sleeplessness state satisfied the criteria of the International Classification of Sleep Disorders (second edition). The exclusion criteria were: 1) poor glycemic control (HbAlc $\geq 8.0 \%), 2$ ) change in the $\mathrm{HbA} 1 \mathrm{c}$ level $\geq 0.5 \%$ in the past 3 months, 3) hepatic failure, 4) treatment for depression, 5) age $<20$ years, 6) history of sleep medication, 7) shift work, 8) treatment for sleep apnea syndrome, and 9) physician's designation as inappropriate for inclusion. As no previous trials have reported the effects of ramelteon on glucose metabolism and sleep quality, we could not calculate the sample size. Most type 2 diabetic patients with insomnia who regularly visited the hospital were already using a sleep medication, and we expected the target sample size of 50 subjects.

\section{Methods}

We analyzed the effect of administration and discontinuation of ramelteon. All of the patients who signed the informed consent were prescribed $8 \mathrm{mg}$ of ramelteon to be taken every night for 3 months. And then patients were divided, by random permuted blocks, into the continuation group that took ramelteon and the discontinuation group that discontinued taking ramelteon for 3 additional months. We defined the first 3 months as the first period and the second 3 months as the second period. During the first period, there was no change or addition of drugs for the treatment of diabetes. In case of exacerbation of insomnia in the discontinuation group, patients would receive a sleep medication, including ramelteon, and be excluded from the analysis of data.

Blood samples and questionnaires were obtained three times, before and after each 3-month period. The primary endpoint was changes in HbAlc level at the end of the each administration period. The secondary endpoints were as follows: changes of other glucose metabolism markers including fasting plasma glucose (FPG), immunoreactive insulin (IRI), serum C-reactive peptide ( $\mathrm{s}-\mathrm{CPR}$ ), lipid metabolism including total cholesterol (TC), low-density lipoprotein cholesterol (LDL-C), high-density lipoprotein cholesterol (HDL-C), and triglycerides (TG), hepatic function parameters, inflammation markers including ferritin, high-sensitivity C-reactive protein (hs-CRP), sleep quality, and health-related quality of life (QOL). Sleep quality was assessed using the Pittsburgh sleep questionnaire index (PSQI). Health-related QOL was assessed using short form 36 (SF-36). Safety was assessed on the basis of all and blood parameters.

\section{Biochemical measurements}

Blood samples were obtained after overnight fasting. HbA1c was measured by HPLC (Adams Alc HA-8160; Arkray Inc., Kyoto, Japan) and plasma glucose was measured using the glucose oxidation method (chemical reagent and Glucose AUTO, and STAT GA-1160 analyzer; Arkray Inc.). TC, LDL$\mathrm{C}$, HDL-C, and TG were measured by standard enzymatic methods (Kyowa Medex, Tokyo, Japan). Glycated albumin (GA), IRI, s-CPR, ferritin, and hs-CRP were measured by SRL Central Laboratory Testing for Clinical Trials (SRL, Inc., Tokyo, Japan).

Insulin resistance was estimated by the modified homeostatic model of assessment of insulin resistance (HOMA-IR) and the quantitative insulin sensitivity check index (QUICKI). Beta cell function was estimated by the CPR index. These indexes were calculated according to the following formula [1922]: HOMA-IR $=$ IRI $(\mu \mathrm{U} / \mathrm{mL}) \times$ FPG $(\mathrm{mg} / \mathrm{dL}) / 405$. QUICKI $=1 /(\log$ fasting insulin $(\mu \mathrm{U} / \mathrm{mL})+\log$ fasting glucose $(\mathrm{mg} /$ $\mathrm{dL})$ ). CPR index $=$ fasting CPR $(\mathrm{ng} / \mathrm{mL}) / \mathrm{FPG}(\mathrm{mg} / \mathrm{dL}) \times 100$.

We excluded patients with an FPG $>140 \mathrm{mg} / \mathrm{dL}$ to calculate the HOMA-IR. We defined insulin resistance as HOMA$\mathrm{IR} \geq 2.5$ and $\mathrm{QUICKI} \leq 0.337$.

\section{PSQI}

Sleep quality was measured using PSQI. PSQI is a self-rated questionnaire containing seven components: sleep quality, sleep latency, sleep duration, sleep efficiency, sleep disturbance, use of sleep medication, and daytime dysfunction [23]. Each component is scored on a range of 0 - 3, for a possible total score range of 0 - 21 (global PSQI score). A higher global PSQI score indicates poorer sleep quality. A global score of $>$ 5 is indicative of poor sleep quality. As this study was to assess the effect of ramelteon intervention in type 2 diabetic patients with insomnia who did not use any sleep medication, we excluded question component 6: use of sleep medication of the PSQI [24, 25].

\section{SF-36}

Subjective health-related QOL was measured using SF-36. SF-36 is a self-rated questionnaire containing 36 items that measure eight health concepts. These health concepts include 


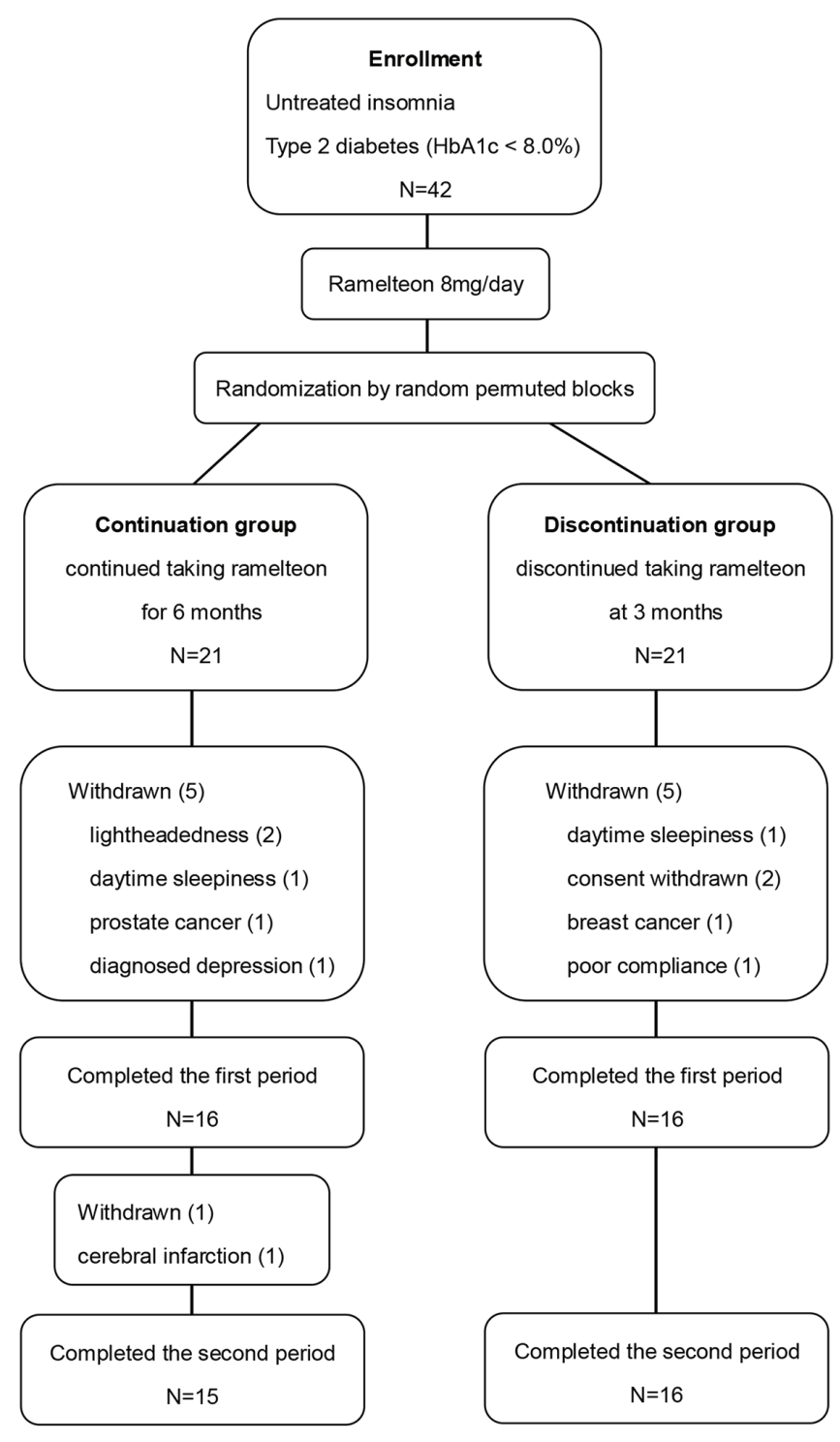

Figure 1. Diagram of patient flow.

physical functioning, role functioning-physical, bodily pain, general health perceptions, vitality, social role functioning, role functioning-emotional, and mental health [26, 27]. Three summary scores, i.e., physical component summary (PCS), mental component summary (MCS), and role/social component summary (RCS), were calculated from the scores of these eight components using a designated calculation program [28]. Each component has a possible score range of 0 - 100 points, with a median value of 50 points.

\section{Statistical analysis}

Statistical analyses were performed using SPSS version 11.0.1 J (SPSS Inc., Armonk, NY, USA). Results were expressed as mean \pm SD. Differences in continuous variables within each group were compared using the paired $t$-test when the vari- able showed a normal distribution, or Wilcoxonsigned-rank test when it did not. Differences in continuous variables between the two groups were compared using the unpaired $t$-test and two-way analysis of variance (ANOVA) when the variable showed a normal distribution or Mann-Whitney's U test when it did not. Categorical variables were compared between the two groups using the Chi-square test. The relations between values at baseline and those at the end of each study period were evaluated using Pearson's rank correlation when the variable showed a normal distribution or Spearman's rank correlation when it did not. The level of statistical significance was set at $\mathrm{P}<0.05$.

\section{Results}

Details of enrolled subjects are shown in Figure 1. Of the 42 patients enrolled, 31 patients completed all control visits during the study. Eleven patients discontinued the study because of the following reasons: two patients suffered lightheadedness and two patients suffered daytime sleepiness when taking ramelteon, both of which subsided after discontinuation of ramelteon, two patients withdrew their informed consent before the start of the administration of ramelteon, one patient was diagnosed with breast cancer and another was diagnosed with prostate cancer soon after giving their informed consent, one patient was diagnosed with depression after registration, and one patient was excluded due to poor treatment compliance. In continuation group, one patient had a medical history of paroxysmal atrial fibrillation and withdrew from the study because of cardiogenic cerebral infarction 4 months later. Therefore, we analyzed the effect of ramelteon in 32 patients in the first period and 31 patients in the second period.

The patients' baseline characteristics are shown in Table 1. Seventeen of the 32 patients were men, with a mean age of 68.9 years old, body mass index (BMI) of $25.7 \mathrm{~kg} / \mathrm{m}^{2}$, diabetes duration of 9.8 years, and mean $\mathrm{HbA} 1 \mathrm{c}$ of $6.7 \%$. Four patients were not treated with hypoglycemic agents. Sulfonylureas, biguanides, thiazolidines, incretin-based drugs and insulin were used by 11, 16, five, 16 and three patients, respectively (some of them used more than one drug). Eighteen patients used antihypertensive agents, and 20 patients used lipid-lowering drugs.

The changes of all clinical parameters in the first period are shown in Table 2. No changes were observed in HbA1c. In addition, no changes were observed in GA, FPG, IRI, s-CPR, CPR index, HOMA-IR, and QUICKI. No factor was found to correlate with the change in HbA1c after ramelteon treatment. Moreover, although we performed subgroup analyses using the baseline values or the percentage change of $\mathrm{HbAlc}, \mathrm{CPR}$ index, HOMA-IR, QUICKI, and PSQI, no effect of ramelteon on the evaluated parameters was observed.

Regarding sleep quality, the global PSQI score improved from 8.1 to 7.2 points $(\mathrm{P}=0.030)$. The scores of sleep quality (C1) and sleep latency (C2) among PSQI components also improved significantly from 1.6 to 1.3 points $(P=0.010)$ and from 1.7 to 1.2 points $(\mathrm{P}=0.010)$, respectively. In fact, time of sleep latency decreased from 23.6 to $18.8 \mathrm{~min}(\mathrm{P}=0.039)$. A correlation was observed between the baseline global PSQI 
Table 1. Baseline Characteristics

\begin{tabular}{|c|c|}
\hline Age (years) & $68.9 \pm 8.1$ \\
\hline Gender (male/female) & $17 / 15$ \\
\hline Height $(\mathrm{cm})$ & $162.3 \pm 9.3$ \\
\hline Body weight (kg) & $68.1 \pm 14.5$ \\
\hline $\operatorname{BMI}\left(\mathrm{kg} / \mathrm{m}^{2}\right)$ & $25.7 \pm 4.3$ \\
\hline Diabetes duration (years) & $9.8 \pm 3.5$ \\
\hline HbA1c $(\%)$ & $6.7 \pm 0.4$ \\
\hline \multicolumn{2}{|l|}{ Medications } \\
\hline Anti-diabetes $(\%)$ & $28(88)$ \\
\hline Sulfonylureas $(\%)$ & $11(34)$ \\
\hline Biguanides $(\%)$ & $16(50)$ \\
\hline Thiazolidines $(\%)$ & $5(16)$ \\
\hline Incretin-based drugs (\%) & $16(50)$ \\
\hline Insulin $(\%)$ & $3(9)$ \\
\hline Anti-hypertension (\%) & $18(56)$ \\
\hline Angiotensin II receptor blockers (\%) & $15(47)$ \\
\hline Calcium channel blockers (\%) & $13(41)$ \\
\hline$\beta$-blockers (\%) & $5(16)$ \\
\hline Anti-dyslipidemia (\%) & $20(63)$ \\
\hline Statins $(\%)$ & $18(56)$ \\
\hline Fibrates $(\%)$ & $2(6)$ \\
\hline Ethyl icosapentate (\%) & $3(9)$ \\
\hline
\end{tabular}

Data are expressed as the mean \pm SD or number (\%). BMI: body mass index.

score and the changing rate of this score $(\mathrm{R}=-0.588, \mathrm{P}=0.001$ in Fig. 2); specifically, a greater improvement in sleep quality was observed in patients with poor sleep quality. On the other hand, no changes in MCS, PCS, and RCS components of the SF-36 were observed.

Regarding lipid metabolism, only the LDL-C level decreased slightly from 105 to $100 \mathrm{mg} / \mathrm{dL}(\mathrm{P}=0.048)$. No changes were observed in hepatic function parameters, inflammation markers or physiological findings, including blood pressure, body weight, and BMI.

The values of all clinical parameters obtained in the second period are shown in Table 3. At the end of the first 3 months, no differences were observed in their medications, physiological findings, glucose metabolism, lipid metabolism, hepatic function, and inflammation marker levels in each group. Similarly, no differences were observed in the scores of global PSQI and PSQI components and SF-36 components.

The level of $\mathrm{HbA} 1 \mathrm{c}$ remained at $6.8 \%$ in the continuation group ( $\mathrm{P}=0.702)$, whereas it increased from $6.7 \%$ to $6.9 \%$ in the discontinuation group $(\mathrm{P}=0.003)$. There was slight difference in change of the HbA1c level by two-way ANOVA between the continuation group and the discontinuation group $(P=0.047)$. Similarly, the level of GA almost did not change in the continuation group $(17.1-17.3 \% ; \mathrm{P}=0.672)$ and it increased from $17.1 \%$ to $17.6 \%$ in the discontinuation group $(\mathrm{P}=$
0.027). But, there was no difference in change of the GA level by two-way ANOVA between the continuation group and the discontinuation group $(\mathrm{P}=0.539)$. No differences were observed in other glucose parameters.

Regarding sleep quality, no change in the global PSQI score was observed in continuation and discontinuation groups during the second period in each group $(\mathrm{P}=0.69$ and $\mathrm{P}=0.32$, respectively). Similarly, no changes were observed in the PSQI components and the score of any SF-36 component. There were no rebound insomnia due to discontinuation of ramelteon, and none of the patients required sleep medication including ramelteon.

No differences were observed in physiological findings, lipid metabolism, hepatic function, and inflammation marker levels.

\section{Discussion}

This is the first study to investigate the effect of ramelteon on glucose metabolism and sleep quality in type 2 diabetic patients with untreated insomnia.

\section{Effect on glucose metabolism}

The primary objective of this study was to investigate the effect of ramelteon on glucose metabolism. In this study, taking ramelteon for 3 or 6 months did not change the level of $\mathrm{HbAlc}$, but discontinuing taking ramelteon for 3 months increased the level of $\mathrm{HbA} 1 \mathrm{c}$ from $6.7 \%$ to $6.9 \%$.

Ramelteon is an agonist of melatonin. The mechanisms of action of melatonin with respect to glucose metabolism have been investigated in various basic studies. As a result, it has been shown that melatonin reduces both blood glucose levels and insulin secretion. Melatonin induces GLUT-4 expression in skeletal muscles through an IRS-1 or PI-3 kinase-mediated pathway and enhances glucose transportation [29, 30]. Melatonin binds to MT1 and MT2 receptors on pancreatic $\beta$ cells, and reduces cAMP and cGMP levels, respectively [17], thereby inhibiting insulin secretion. In diabetic rats, it was also shown that melatonin improves insulin resistance by inhibiting inflammatory cytokine production and oxidative stress and reducing the level of free fatty acids $[12,31]$.

In addition to those basic studies, it has been reported that melatonin improves glucose metabolism in patients with type 2 diabetes. Hussain et al observed a decrease of $\mathrm{HbAlc}$ from $7.63 \%$ to $7.13 \%$ in patients after 3 months on high dose of melatonin [13]. Besides, Garfinkel et al reported a decrease of $\mathrm{HbA} 1 \mathrm{c}$ from $9.13 \%$ to $8.47 \%$ in patients who took prolongedrelease melatonin for 5 months [14].

Regarding the effect of a sleep intervention on glucose metabolism, it was reported that treatment with $5-10 \mathrm{mg}$ of zolpidem for 3 months significantly improved the $\mathrm{HbA} 1 \mathrm{c}$ level by $0.4 \%$ from $6.5 \%$ in 14 Japanese patients with type 2 diabetes complicated by insomnia [32]. Sleep improvement of glucose metabolism might be mediated by sympathetic nerve's activity. 
Table 2. Effects of Ramelteon in the First Period

\begin{tabular}{|c|c|c|c|}
\hline & Baseline & 3 months & P value \\
\hline Gender (male/female) & $17 / 15$ & & \\
\hline Body weight (kg) & $68.1 \pm 14.5$ & $68.4 \pm 14.4$ & \\
\hline BMI $\left(\mathrm{kg} / \mathrm{m}^{2}\right)$ & $25.7 \pm 4.3$ & $25.8 \pm 4.3$ & \\
\hline Systolic blood pressure (mm Hg) & $138 \pm 21$ & $135 \pm 20$ & \\
\hline Diastolic blood pressure $(\mathrm{mm} \mathrm{Hg})$ & $79 \pm 11$ & $81 \pm 11$ & \\
\hline $\mathrm{HbAlc}(\%)$ & $6.7 \pm 0.4$ & $6.7 \pm 0.5$ & \\
\hline GA $(\%)$ & $17.1 \pm 2.6$ & $17.2 \pm 2.4$ & \\
\hline FPG (mg/dL) & $120 \pm 18$ & $125 \pm 23$ & \\
\hline IRI $(\mu \mathrm{U} / \mathrm{mL})$ & $8.01 \pm 6.80$ & $8.01 \pm 7.30$ & \\
\hline s-CPR (ng/mL) & $2.19 \pm 1.23$ & $2.12 \pm 1.16$ & \\
\hline HOMA-IR & $2.08 \pm 1.59$ & $1.92 \pm 1.40$ & \\
\hline QUICKI & $0.35 \pm 0.04$ & $0.35 \pm 0.04$ & \\
\hline CPR index & $1.84 \pm 1.01$ & $1.72 \pm 0.96$ & \\
\hline Total cholesterol (mg/dL) & $177 \pm 33$ & $171 \pm 29$ & \\
\hline LDL-C (mg/dL) & $105 \pm 27$ & $100 \pm 24$ & 0.048 \\
\hline HDL-C (mg/dL) & $60 \pm 17$ & $58 \pm 15$ & \\
\hline LDL-C/HDL-C & $1.9 \pm 0.6$ & $1.8 \pm 0.6$ & \\
\hline Triglyceride (mg/dL) & $131 \pm 76$ & $117 \pm 63$ & \\
\hline Non-HDL-C (mg/dL) & $117 \pm 29$ & $112 \pm 27$ & \\
\hline Cre $(\mathrm{mg} / \mathrm{dL})$ & $0.82 \pm 0.22$ & $0.83 \pm 0.23$ & \\
\hline eGFR (mL/min) & $65.4 \pm 13.4$ & $65.6 \pm 14.0$ & \\
\hline UAE (mg/g.Cre) & $28.6 \pm 49.6$ & $42.8 \pm 87.3$ & \\
\hline $\operatorname{AST}(\mathrm{U} / \mathrm{L})$ & $24 \pm 8$ & $25 \pm 9$ & \\
\hline $\operatorname{ALT}(\mathrm{U} / \mathrm{L})$ & $25 \pm 13$ & $25 \pm 9$ & \\
\hline$\gamma \mathrm{GTP}(\mathrm{U} / \mathrm{L})$ & $30 \pm 16$ & $30 \pm 16$ & \\
\hline Ferritin $(\mathrm{ng} / \mathrm{mL})$ & $140 \pm 149$ & $127 \pm 102$ & \\
\hline hs-CRP (ng/mL) & $1,060 \pm 2,358$ & $1,383 \pm 2,569$ & \\
\hline PSQI $(0$ - 18) & $8.1 \pm 4.1$ & $7.2 \pm 2.8$ & 0.030 \\
\hline $\mathrm{C} 1(0-3)$ & $1.6 \pm 0.8$ & $1.3 \pm 0.7$ & 0.010 \\
\hline $\mathrm{C} 2(0-3)$ & $1.7 \pm 2.1$ & $1.2 \pm 1.8$ & 0.040 \\
\hline $\mathrm{C} 3(0-3)$ & $1.8 \pm 1.0$ & $1.6 \pm 0.9$ & \\
\hline $\mathrm{C} 4(0-3)$ & $0.8 \pm 1.0$ & $0.8 \pm 1.0$ & \\
\hline $\mathrm{C} 5(0-3)$ & $1.0 \pm 0.4$ & $0.9 \pm 0.3$ & \\
\hline $\mathrm{C} 7(0-3)$ & $1.5 \pm 1.1$ & $1.5 \pm 0.8$ & \\
\hline Sleep latency (min) & $23.6 \pm 25.7$ & $18.8 \pm 17.6$ & 0.039 \\
\hline Total sleep time (h) & $6.1 \pm 2.4$ & $6.2 \pm 2.1$ & \\
\hline \multicolumn{4}{|l|}{ SF-36 } \\
\hline $\operatorname{PCS}(0-100)$ & $47.3 \pm 13.5$ & $50.1 \pm 12.3$ & \\
\hline $\operatorname{MCS}(0-100)$ & $54.1 \pm 9.7$ & $53.3 \pm 8.7$ & \\
\hline $\operatorname{RCS}(0-100)$ & $36.7 \pm 10.5$ & $38.5 \pm 8.6$ & \\
\hline
\end{tabular}

Data are expressed as the mean \pm SD or number (\%). BMI: body mass index; GA: glycated albumin; FPG: fasting plasma glucose; IRI: immunoreactive insulin; s-CPR: serum C-peptide immunoreactivity; HOMA-IR: homeostatic model of assessment of insulin resistance; QUICKI: quantitative insulin sensitivity check index; LDL-C: low-density lipoprotein cholesterol; HDL-C: high-density lipoprotein cholesterol; eGFR: estimated glomerular filtration rate; UAE: urinary albumin excretion; hs-CRP: high-sensitivity C-reactive protein; PSQI: Pittsburgh sleep questionnaire index; SF-36: short form 36; PCS: physical component summary; MCS: mental component summary; RCS: role/social component summary. 


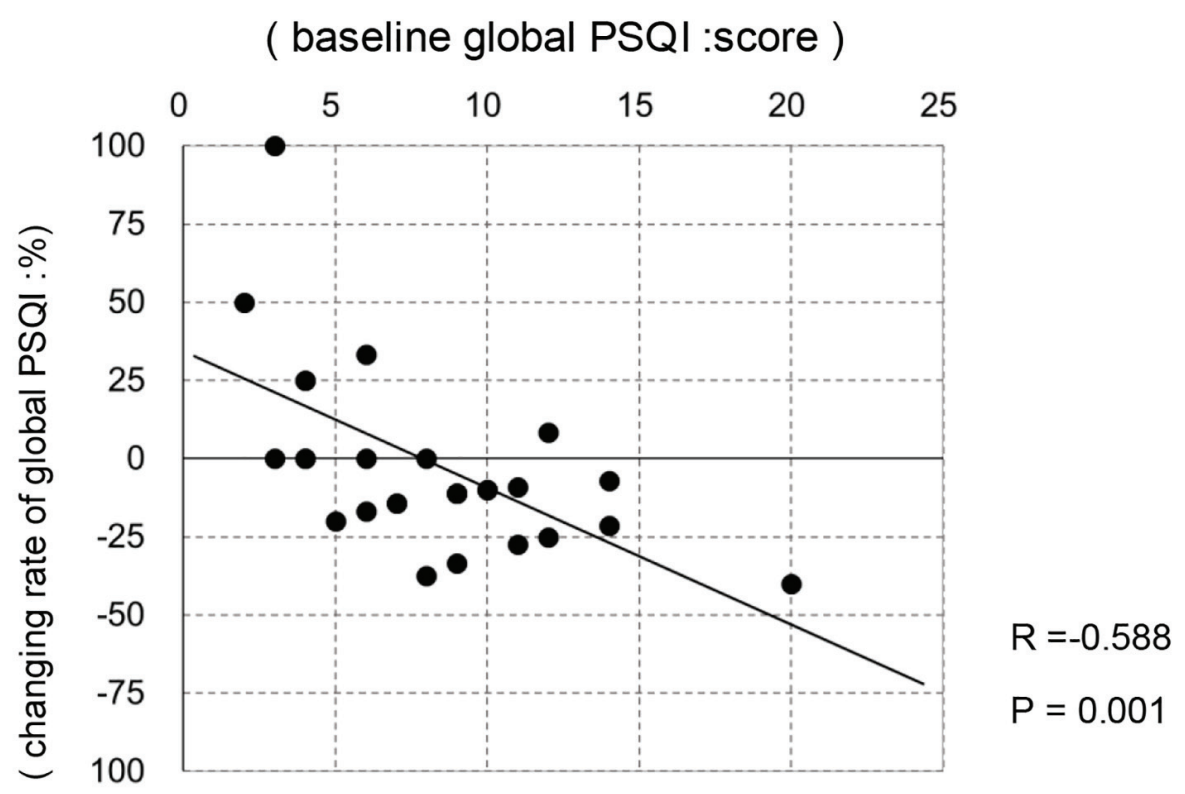

Figure 2. The correlation between baseline global PSQI and changing rate of global PSQI in the first period.

The level of HbA1c was not changed by ramelteon; however, the level of HbA1c increased after discontinuation of ramelteon. The level of $\mathrm{HbA} 1 \mathrm{c}$ in patients with type 2 diabetes increases during the natural course of the disease or due to seasonal change even if for a short term [33-38]. Although we could not set a placebo group, the increase of HbA1c in the discontinuation group was possibly the result of the natural course of type 2 diabetes or of seasonal change. There has been no previous report on the potential effects of ramelteon on glucose metabolism. The subjects in two previous reports about melatonin were administered either a high dose of melatonin $(10 \mathrm{mg})$ or prolonged-release melatonin with a half-life of 8 $\mathrm{h}[13,14]$. The mean HbAlc level attained in these studies was $7.63 \%$ and $9.13 \%$, respectively and with poor glycemic control. In contrast, ramelteon is a melatonin receptor agonist, not melatonin itself, and the half-life of ramelteon is as short as $1 \mathrm{~h} \mathrm{[39].} \mathrm{Patients} \mathrm{in} \mathrm{this} \mathrm{study} \mathrm{had} \mathrm{a} \mathrm{relatively} \mathrm{stable} \mathrm{glyce-}$ mic control with a mean HbA1c level of $6.7 \%$. We excluded patients with an HbA1c level of $\geq 8.0 \%$ indicative of poor glycemic control from this study because they had to receive treatment for diabetes prior to a sleep intervention. Therefore, differences in the drugs dosages and half-lives and glycemic control of the study population at baseline might have contributed to the difference in glycemic control improvement between the present study and those previously reported.

\section{Effect on sleep}

The secondary objective of this study was to investigate the effect of ramelteon on sleep quality. In this study, ramelteon improved sleep quality in the type 2 diabetic patients with untreated insomnia. The sleep quality attained with ramelteon has been maintained even after discontinuing ramelteon.
Ramelteon has MT1 and MT2 receptor affinities 5.8-fold and 3.4-fold, which are higher than those of melatonin itself [16]. Ramelteon is not expected to induce rebound insomnia or withdrawal effects, or to cause abuse or addiction [40].

Compared with healthy individuals, patients with type 2 diabetes frequently experience insomnia [1]. One of the reasons might be decreased melatonin secretion. Decreased blood melatonin level and urinary 6-sulfatoxymelatonin level have been observed in diabetic patients [41]. Another reason might be the nocturnal awakening and unrefreshing sleep due to a neurogenic bladder and painful diabetic neuropathy.

Regarding the features of sleep disturbance among patients in this study, we observed difficulty in falling asleep with a mean sleep latency of 23.6 min. Besides, nocturnal awakening onset was often noted as a reason for sleep difficulties. Administration of $8 \mathrm{mg}$ of ramelteon for 3 months reduced sleep latency to $18.8 \mathrm{~min}$, and improved the subjective sleep quality and sleep latency in PSQI component scores and the global PSQI score. The quality of sleep improved by ramelteon has been maintained even after discontinuing taking ramelteon. Many previous studies reported changes in sleep quality after the administration of ramelteon. Sleep quality improved in response to the administration of ramelteon in patients with primary insomnia, gastroesophageal reflux disease, generalized anxiety disorder, and sleep apnea syndrome, and postmenopausal women [40, 42-45]. This is the first study undertaken to assess the effect of ramelteon in patients with type 2 diabetes. The results of this study suggest that ramelteon might be also useful for diabetic patients with insomnia.

The incidence of adverse events associated to the administration of ramelteon was reported to be approximately $10 \%$ [46]. In this study, four of the 34 patients withdrew from the study because of lightheadedness or daytime sleepiness; however, no other adverse events were observed during the study. 
Table 3. Effects of Continuating or Discontinuating Ramelteon in the Second Period

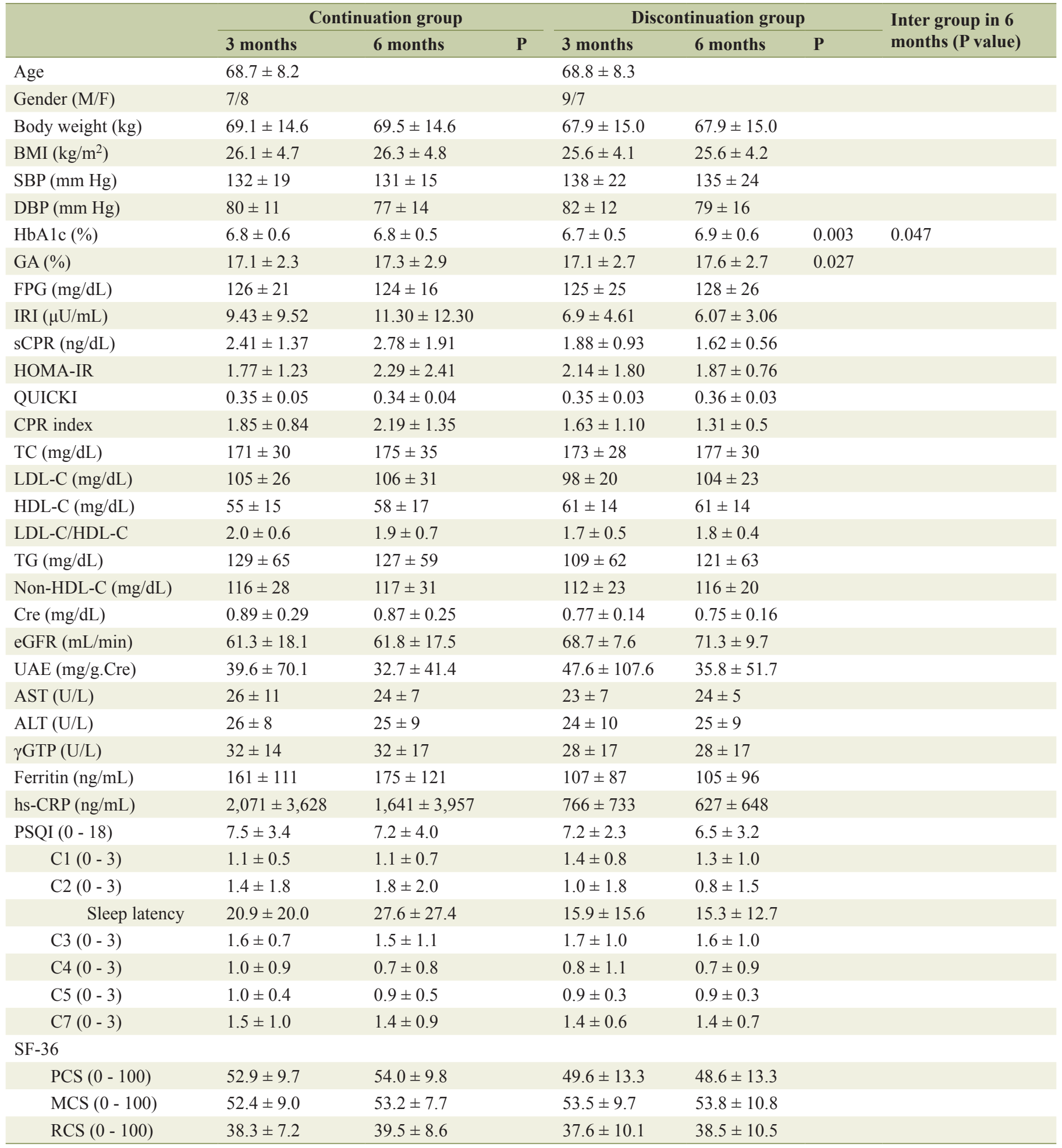

Data are expressed as the mean \pm SD or number (\%). BMI: body mass index; SBP: systolic blood pressure; DBP: diastolic blood pressure; GA: glycated albumin; FPG: fasting plasma glucose; IRI: immunoreactive insulin; s-CPR: serum C-peptide immunoreactivity; HOMA-IR: homeostatic model of assessment of insulin resistance; QUICKI: quantitative insulin sensitivity check index; TC: total cholesterol; LDL-C: Iow-density lipoprotein cholesterol; HDL-C: high-density lipoprotein cholesterol; TG: triglyceride; eGFR: estimated glomerular filtration rate; UAE: urinary albumin excretion; hs-CRP: high-sensitivity C-reactive protein; PSQI: Pittsburgh sleep questionnaire index; SF-36: short form 36; PCS: physical component summary; MCS: mental component summary; RCS: role/social component summary. 
The rate of adverse events did not differ from that found in previous reports.

Although problems such as rebound insomnia, drug withdrawal symptoms, abuse and addiction, have been reported with the use of some sleep medications [47], such problems have not been reported with the use of ramelteon. As for adverse events due to the discontinuation of ramelteon, sleep quality problems did not become exacerbated after discontinuation of ramelteon, and no adverse events such as rebound or withdrawal symptoms were observed either. In other words, discontinuation of ramelteon is unlikely to cause any disadvantage.

\section{Association with health-related QOL}

SF-36 was used in this study as a conventional index of healthrelated QOL. Some studies reported changes of the scores of SF-36 components after the administration of melatonin. Melatonin was reported to improve QOL, as assessed using the SF-36, in patients with primary insomnia and delayed sleep phase syndrome [48, 49], whereas no improvement in the QOL was observed in patients with tetraplegia and chronic fatigue syndrome $[50,51]$. There have been no previous reports on the effects of ramelteon on health-related QOL of patients, regardless of diabetes; accordingly, this is the first report on the effect of ramelteon assessed using SF-36. However, no changes were observed in PCS, MCS, or RCS scores. Although global PSQI score, sleep quality and sleep latency improved, no improvement was observed in other parameters such as total sleep time or sleep efficiency, and therefore this might not have led to improvement in QOL.

\section{Protocol}

As there is no precedent for ramelteon administration to type 2 diabetic patients with untreated insomnia, it was unknown when the beneficial effect of ramelteon on glucose metabolism would appear in this population.

A 6-month observation period was set to prevent the effect of seasonal changes in diet and exercise that would have profoundly affected the results if a longer had been set. In contrast, an observation period of $<6$ months would have not allowed the examination of the effect of discontinuation of ramelteon on $\mathrm{HbA} 1 \mathrm{c}$. To determine this period, we referred to two previous melatonin studies with 3- and 5-month observation periods.

In the present study, all patients were administered ramelteon at the start of the study. For an accurate evaluation of the effect of ramelteon, it would have been preferable to assign patients initially to two groups (administration or discontinuation of ramelteon) and to set a placebo group. However, as patients can subjectively determine the effect of sleep medication, patients in the placebo group may experience a loss of trust in their physicians because they are not experiencing any improvements in sleep. Moreover, patients in the non-ramelteon group might insist on consent withdrawal. Therefore, ramelt- eon was administered to all patients.

\section{Limitations of this study}

This study has some limitations. First, most type 2 diabetic patients with insomnia who regularly visited the hospital were already using a sleep medication. The rest of patients had not complained of or had not been asked from a doctor about sleeplessness. As only a few patients were satisfied with the conditions of untreated chronic insomnia, the number of registered patients was smaller than expected. Second, patients with an $\mathrm{HbA} 1 \mathrm{c}$ level of $\geq 8 \%$ were excluded and thus, few patients exhibited inadequate or poor glycemic control. We expected similar outcomes by administrating ramelteon to patients whose glycemic control was stable. However, ramelteon did not improve glucose metabolism in them. Ramelteon would likely have improved glucose metabolism in patients with a poor glycemic control.

\section{Conclusion}

Treatment with ramelteon did not change the HbA1c level but improved sleep quality in type 2 diabetic patients with insomnia. Discontinuation of ramelteon slightly increased the HbA1c level and did not worsen sleep quality.

\section{References}

1. Gottlieb DJ, Punjabi NM, Newman AB, Resnick HE, Redline S, Baldwin CM, Nieto FJ. Association of sleep time with diabetes mellitus and impaired glucose tolerance. Arch Intern Med. 2005;165(8):863-867.

2. Pyykkonen AJ, Isomaa B, Pesonen AK, Eriksson JG, Groop L, Tuomi T, Raikkonen K. Subjective sleep complaints are associated with insulin resistance in individuals without diabetes: the PPP-Botnia Study. Diabetes Care. 2012;35(11):2271-2278.

3. Taheri S, Lin L, Austin D, Young T, Mignot E. Short sleep duration is associated with reduced leptin, elevated ghrelin, and increased body mass index. PLoS Med. 2004;1(3):e62.

4. Gulec M, Ozkol H, Selvi Y, Tuluce Y, Aydin A, Besiroglu L, Ozdemir PG. Oxidative stress in patients with primary insomnia. Prog Neuropsychopharmacol Biol Psychiatry. 2012;37(2):247-251.

5. Vgontzas AN, Liao D, Pejovic S, Calhoun S, Karataraki $\mathrm{M}$, Bixler EO. Insomnia with objective short sleep duration is associated with type 2 diabetes: A populationbased study. Diabetes Care. 2009;32(11):1980-1985.

6. Papantoniou K, Pozo OJ, Espinosa A, Marcos J, CastanoVinyals G, Basagana X, Ribas FC, et al. Circadian variation of melatonin, light exposure, and diurnal preference in day and night shift workers of both sexes. Cancer Epidemiol Biomarkers Prev. 2014;23(7):1176-1186.

7. Dumont M, Paquet J. Progressive decrease of melatonin production over consecutive days of simulated night 
work. Chronobiol Int. 2014;31(10):1231-1238.

8. Sharma M, Palacios-Bois J, Schwartz G, Iskandar H, Thakur M, Quirion R, Nair NP. Circadian rhythms of melatonin and cortisol in aging. Biol Psychiatry. 1989;25(3):305-319.

9. McMullan CJ, Schernhammer ES, Rimm EB, Hu FB, Forman JP. Melatonin secretion and the incidence of type 2 diabetes. JAMA. 2013;309(13):1388-1396.

10. la Fleur SE, Kalsbeek A, Wortel J, van der Vliet J, Buijs RM. Role for the pineal and melatonin in glucose homeostasis: pinealectomy increases night-time glucose concentrations. J Neuroendocrinol. 2001;13(12):1025-1032.

11. Peschke E, Stumpf I, Bazwinsky I, Litvak L, Dralle H, Muhlbauer E. Melatonin and type 2 diabetes - a possible link? J Pineal Res. 2007;42(4):350-358.

12. Agil A, Rosado I, Ruiz R, Figueroa A, Zen N, Fernandez-Vazquez G. Melatonin improves glucose homeostasis in young Zucker diabetic fatty rats. J Pineal Res. 2012;52(2):203-210.

13. Hussain SA, Khadim HM, Khalaf BH, Ismail SH, Hussein KI, Sahib AS. Effects of melatonin and zinc on glycemic control in type 2 diabetic patients poorly controlled with metformin. Saudi Med J. 2006;27(10):1483-1488.

14. Garfinkel D, Zorin M, Wainstein J, Matas Z, Laudon M, Zisapel N. Efficacy and safety of prolonged-release melatonin in insomnia patients with diabetes: a randomized, double-blind, crossover study. Diabetes Metab Syndr Obes. 2011;4:307-313.

15. Uchikawa O, Fukatsu K, Tokunoh R, Kawada M, Matsumoto K, Imai Y, Hinuma S, et al. Synthesis of a novel series of tricyclic indan derivatives as melatonin receptor agonists. J Med Chem. 2002;45(19):4222-4239.

16. Kato K, Hirai K, Nishiyama K, Uchikawa O, Fukatsu K, Ohkawa S, Kawamata Y, et al. Neurochemical properties of ramelteon (TAK-375), a selective MT1/MT2 receptor agonist. Neuropharmacology. 2005;48(2):301-310.

17. She M, Laudon M, Yin W. Melatonin receptors in diabetes: a potential new therapeutical target? Eur J Pharmacol. 2014;744:220-223.

18. Picinato MC, Haber EP, Cipolla-Neto J, Curi R, de O1iveira Carvalho CR, Carpinelli AR. Melatonin inhibits insulin secretion and decreases PKA levels without interfering with glucose metabolism in rat pancreatic islets. J Pineal Res. 2002;33(3):156-160.

19. Matthews DR, Hosker JP, Rudenski AS, Naylor BA, Treacher DF, Turner RC. Homeostasis model assessment: insulin resistance and beta-cell function from fasting plasma glucose and insulin concentrations in man. Diabetologia. $1985 ; 28(7): 412-419$.

20. Katz A, Nambi SS, Mather K, Baron AD, Follmann DA, Sullivan G, Quon MJ. Quantitative insulin sensitivity check index: a simple, accurate method for assessing insulin sensitivity in humans. J Clin Endocrinol Metab. 2000;85(7):2402-2410.

21. Asano T, Kawamura M, Watanabe T, Abe M, Chin R, Miyazaki S, Hirata Y. Indices of urinary and serum c-peptide corrected with fasting plasma glucose for decisionmaking of insulin therapy in type 2 diabetes-validation and comparison. Journal of Japan Diabetes Society.
2008;51:759-763 (In Japanese).

22. Iwata M, Matsushita Y, Fukuda K, Wakura T, Okabe K, Koshimizu Y, Fukushima Y, et al. Secretory units of islets in transplantation index is a useful predictor of insulin requirement in Japanese type 2 diabetic patients. J Diabetes Investig. 2014;5(5):570-580.

23. Buysse DJ, Reynolds CF, 3rd, Monk TH, Berman SR, Kupfer DJ. The Pittsburgh Sleep Quality Index: a new instrument for psychiatric practice and research. Psychiatry Res. 1989;28(2):193-213.

24. Bourgeois J, Elseviers MM, Van Bortel L, Petrovic M, Vander Stichele RH. Sleep quality of benzodiazepine users in nursing homes: a comparative study with nonusers. Sleep Med. 2013;14(7):614-621.

25. Bourgeois J, Elseviers MM, Van Bortel L, Petrovic M, Vander Stichele RH. Feasibility of discontinuing chronic benzodiazepine use in nursing home residents: a pilot study. Eur J Clin Pharmacol. 2014;70(10):1251-1260.

26. Fukuhara S, Ware JE, Jr., Kosinski M, Wada S, Gandek B. Psychometric and clinical tests of validity of the Japanese SF-36 Health Survey. J Clin Epidemiol. 1998;51(11):1045-1053.

27. Fukuhara S, Bito S, Green J, Hsiao A, Kurokawa K. Translation, adaptation, and validation of the SF-36 Health Survey for use in Japan. J Clin Epidemiol. 1998;51(11):1037-1044.

28. Suzukamo Y, Fukuhara S, Green J, Kosinski M, Gandek B, Ware JE. Validation testing of a three-component model of Short Form-36 scores. J Clin Epidemiol. 2011;64(3):301-308.

29. She M, Hou H, Wang Z, Zhang C, Laudon M, Yin W. Melatonin rescues 3T3-L1 adipocytes from FFA-induced insulin resistance by inhibiting phosphorylation of IRS-1 on Ser307. Biochimie. 2014;103:126-130.

30. Ha E, Yim SV, Chung JH, Yoon KS, Kang I, Cho YH, Baik HH. Melatonin stimulates glucose transport via insulin receptor substrate-1/phosphatidylinositol 3-kinase pathway in C2C12 murine skeletal muscle cells. J Pineal Res. 2006;41(1):67-72.

31. Agil A, Reiter RJ, Jimenez-Aranda A, Iban-Arias R, Navarro-Alarcon M, Marchal JA, Adem A, et al. Melatonin ameliorates low-grade inflammation and oxidative stress in young Zucker diabetic fatty rats. J Pineal Res. 2013;54(4):381-388.

32. Nagata K, Hirokado Y, Otsuki T. Sleep disorder and control of lifestyle related disease. Japanese journal of sleep medicine. 2009;3:85-91 (In Japanese).

33. Aronoff S, Rosenblatt S, Braithwaite S, Egan JW, Mathisen AL, Schneider RL. Pioglitazone hydrochloride monotherapy improves glycemic control in the treatment of patients with type 2 diabetes: a 6-month randomized placebo-controlled dose-response study. The Pioglitazone 001 Study Group. Diabetes Care. 2000;23(11):1605-1611.

34. Kashiwagi A, Kazuta K, Yoshida S, Nagase I. Randomized, placebo-controlled, double-blind glycemic control trial of novel sodium-dependent glucose cotransporter 2 inhibitor ipragliflozin in Japanese patients with type 2 diabetes mellitus. J Diabetes Investig. 2014;5(4):382391. 
35. Yang HK, Min KW, Park SW, Chung CH, Park KS, Choi $\mathrm{SH}$, Song KH, et al. A randomized, placebo-controlled, double-blind, phase 3 trial to evaluate the efficacy and safety of anagliptin in drug-naive patients with type 2 diabetes. Endocr J. 2015;62(5):449-462.

36. Ishii H, Suzuki H, Baba T, Nakamura K, Watanabe T. Seasonal variation of glycemic control in type 2 diabetic patients. Diabetes Care. 2001;24(8):1503.

37. Chen HS, Jap TS, Chen RL, Lin HD. A prospective study of glycemic control during holiday time in type 2 diabetic patients. Diabetes Care. 2004;27(2):326-330.

38. Sohmiya M, Kanazawa I, Kato Y. Seasonal changes in body composition and blood HbAlc levels without weight change in male patients with type 2 diabetes treated with insulin. Diabetes Care. 2004;27(5):1238-1239.

39. Karim A, Tolbert D, Cao C. Disposition kinetics and tolerance of escalating single doses of ramelteon, a highaffinity MT1 and MT2 melatonin receptor agonist indicated for treatment of insomnia. J Clin Pharmacol. 2006;46(2):140-148.

40. Uchiyama M, Hamamura M, Kuwano T, Nishiyama H, Nagata H, Uchimura N. Evaluation of subjective efficacy and safety of ramelteon in Japanese subjects with chronic insomnia. Sleep Med. 2011;12(2):119-126.

41. Obayashi K, Saeki K, Iwamoto J, Ikada Y, Kurumatani N. Independent associations of exposure to evening light and nocturnal urinary melatonin excretion with diabetes in the elderly. Chronobiol Int. 2014;31(3):394-400.

42. Jha LK, Fass R, Gadam R, Maradey-Romero C, Nasrollah L, Hershcovici T, Quan SF, et al. The Effect of Ramelteon on Heartburn Symptoms of Patients With Gastroesophageal Reflux Disease and Chronic Insomnia: A Pilot Study. J Clin Gastroenterol. 2016;50(2):e19-24.

43. Gross PK, Nourse R, Wasser TE. Ramelteon for insomnia symptoms in a community sample of adults with general- ized anxiety disorder: an open label study. J Clin Sleep Med. 2009;5(1):28-33.

44. Gooneratne NS, Gehrman P, Gurubhagavatula I, Al-Shehabi E, Marie E, Schwab R. Effectiveness of ramelteon for insomnia symptoms in older adults with obstructive sleep apnea: a randomized placebo-controlled pilot study. J Clin Sleep Med. 2010;6(6):572-580.

45. Dobkin RD, Menza M, Bienfait KL, Allen LA, Marin H, Gara MA. Ramelteon for the treatment of insomnia in menopausal women. Menopause Int. 2009;15(1):13-18.

46. Uchiyama M, Hamamura $M$, Kuwano $T$, Nagata $H$, Hashimoto T, Ogawa A, Uchimura N. Long-term safety and efficacy of ramelteon in Japanese patients with chronic insomnia. Sleep Med. 2011;12(2):127-133.

47. Gillin JC, Spinweber CL, Johnson LC. Rebound insomnia: a critical review. J Clin Psychopharmacol. 1989;9(3):161-172.

48. Rondanelli M, Opizzi A, Monteferrario F, Antoniello N, Manni R, Klersy C. The effect of melatonin, magnesium, and zinc on primary insomnia in long-term care facility residents in Italy: a double-blind, placebo-controlled clinical trial. J Am Geriatr Soc. 2011;59(1):82-90.

49. Nagtegaal JE, Laurant MW, Kerkhof GA, Smits MG, van der Meer YG, Coenen AM. Effects of melatonin on the quality of life in patients with delayed sleep phase syndrome. J Psychosom Res. 2000;48(1):45-50.

50. Zeitzer JM, Ku B, Ota D, Kiratli BJ. Randomized controlled trial of pharmacological replacement of melatonin for sleep disruption in individuals with tetraplegia. J Spinal Cord Med. 2014;37(1):46-53.

51. Williams G, Waterhouse J, Mugarza J, Minors D, Hayden $\mathrm{K}$. Therapy of circadian rhythm disorders in chronic fatigue syndrome: no symptomatic improvement with melatonin or phototherapy. Eur J Clin Invest. 2002;32(11):831837. 